\title{
Designing nanoparticles as glues for hydrogels: insights from a microscopic model
}

\author{
Nicola Molinari, ${ }^{*, \dagger,+,}$ Guwon Jung, ${ }^{\S, \|}$ and Stefano Angioletti-Uberti*, ${ }^{*}, \boldsymbol{\Phi}$ \\ $\dagger$ †ohn A. Paulson School of Engineering and Applied Sciences, Harvard University, \\ Cambridge, MA 02138, USA \\ $\ddagger$ Department of Physics, Imperial College London, London SWr 2AZ, UK \\ \Thomas Young Centre for Theory and Simulation of Materials, Imperial College London, \\ London SWr 2AZ, UK \\ $\S$ Department of Materials, Imperial College London, London SW' 2AZ, UK \\ ॥Cavendish Laboratory, University of Cambridge, Cambridge, CB3 OHE, UK \\ E-mail: nmolinari@seas.harvard.edu; sangiole@imperial.ac.uk
}




\title{
Graphical TOC entry
}

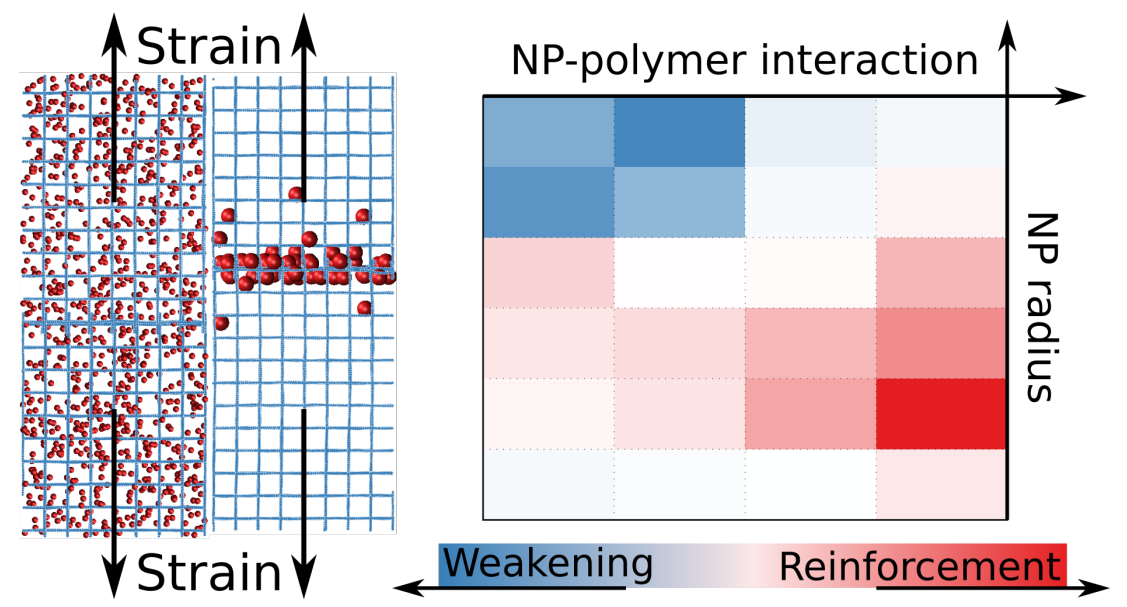

\begin{abstract}
Solution of nanoparticles can be used as a general platform to design glues for polymer-based gels. However, optimisation of the system for a specific gel type requires understanding how different experimentally tuneable parameters concur to determine the gel-gel interfacial adhesion. Here we use molecular dynamics simulations to investigate the effect of nanoparticle size and gel-nanoparticle interaction for a coarse-grained model representative of hydrogels, or in other words a cross-linked polymer network in a swollen state, of varying stiffness. We show that, regardless of the stiffness, competing effects lead to an optimal nanoparticle size for interface reinforcement that is always around the polymer mesh size. We also show that moving away from this optimal size the glue performance can quickly degrade, to the point that nanoparticles can even weaken interface adhesion, suggesting a limit to the amount of polydispersity in the particle size that can be accepted to obtain a functional glue. Overall, these simulations provide a further step towards the rational design of nanoparticles solutions with optimal gluing properties.
\end{abstract}




\section{Introduction}

In recent years, solutions of nanoparticles have been shown to be a viable candidate for the development of glues for cross-linked polymer gels, 1 115 a notoriously difficult technological problem. ${ }^{6}$ One of the appealing properties of this system is that, contrary to other types of glues, no reaction is required to occur between the nanoparticle and the polymer because adhesion is determined by the formation of weak-but-numerous, i.e., reversible, bonds between the nanoparticle surface and the gel. This represents an advantage when the polymer to be glued is of biological origins, e.g., because no activation by exposition to UV-light or via other external stimuli is necessary, avoiding potential damage and thus toxicity of the glue. Moreover, from a mechanical perspective binding via reversible weak bonds provides a mechanism for stress release that increases interface toughness while also avoiding fragile rupture.1 At the same time, the reversibility of these physical bonds means that nanoparticles-based glues possesses self-healing property, improving their mechanical properties under cyclic stress conditions.

Given the aforementioned premises, it should not come as a surprise that one of the major applications of this system is in the development of glues for biological tissues. This is because one of the main components of most tissues is the so-called extra cellular matrix (ECM), whose main structural component in chemical terms can be seen as a cross-linked hydrogel. ${ }^{78}$ Although in general it is made of hydrophilic polymers, the extra cellular matrix exhibits a strong variation in mechanical properties depending on the type of tissue involved, ${ }^{79}$ a variation that might be necessary to consider when designing a glue for a specific tissue. In this design step, various parameters can be controlled experimentally, in particular, the nanoparticle surface chemistry and size, as well as the nanoparticle shape. Although the latter plays an important role and will be discussed in a future publication, here we focus on the systematic study of the first two parameters. We perform molecular dynamics simulations with a coarse-grained model that is adapted from the approach previously adopted in ${ }^{4 \mid 10}$ for nanoparticles interacting with a cross-linked polymer network in 
the collapsed state. The model in $\frac{410}{40}$ is expanded to include two additional angular terms in the potential energy, one for the edges and one for the corners of the polymer network. As previously shown, 11 these additional terms to the potential energy of the system provide a simple and effective way to simulate polymer networks in good solvent, i.e., in the swollen state. This state arises from a competition between chain-chain affinity (which favours a collapsed gel) and stiffness (which favours a swollen state). Because the angular terms allow us to tune the gel stiffness, which grows monotonically as the angular term increases, above a certain threshold dependent on the chain-chain affinity (see Figure 1) a collapsed-to-swollen transition occurs. Studying the nanoparticles-mediated adhesion of polymer networks that achieve this swollen state in different ways (different combinations of chain-chain affinity and stiffness) allows us to uncover robust trends, i.e., trends that do not depend on the specifics of how the swollen state is achieved. This is particularly important because it means our conclusions should be robust or, in other words, not dependent on the exact details of the swollen gel,, 12 and thus be widely observable in experiments. In particular, in this work we want to focus our efforts on answering a relatively simple question, whose answer nevertheless is critical for applications: what is the optimal nanoparticle size that provides the highest possible interface adhesion between two hydrogels (an archetypal swollen gel), and how does this size depend on the state of the gel, if it does at all? In the seminal paper from Rose et al. reporting about nanoparticles-based solutions as glues for hydrogels, they speculated that this size should be close to the mesh size. However, to the best of our knowledge, no systematic study of such effects has appeared in the literature, neither experimentally nor computationally. We aim to close this gap, as well as to provide insights into the microscopic mechanism for the presence of an optimal value.

The reminder of this paper is organised as follows. First, we briefly summarise the computational approach used in Ref. ${ }^{4}$ for studying nanoparticles at the interface between two gels in the collapsed state. We then describe how we modified this coarse-grained model to specifically investigate hydrogels, i.e. swollen gels, with different network stiffness. Then 
we present and critically discuss the results of a systematic set of simulations used to span different combination of parameters of the system, trying to elucidate the physical mechanisms behind the observed behaviour. Furthermore, we also discuss their consequences for the practical design of nanoparticles solutions for hydrogels, taking care to point out in which way the limitations intrinsic in our coarse-grained description might affect our claims. Finally, we draw our conclusions and provide directions for further studies.

\section{Methods}

\section{Structure Generation}

The structures we used in our simulations are generated according to the following procedure. Similarly to what previously done in, ${ }^{4}$ we represent the gel as a series of connected and interacting beads. Polymer beads are placed on the edges of a 3-dimensional cubic grids with side length equal to $11.0 \sigma$, Figure 1(a). The beads are positioned at a distance of $1.0 \sigma$ apart along an edge, which corresponds to the equilibrium position of the harmonic bond potential, as it will be discussed later. As the radius of a single polymer bead is set to $0.5 \sigma$, the effective "empty" face of the cubic grid is $10.0 \sigma$ in all directions. At this stage of the generation, the whole simulation box comprises a total of 41664 beads; the number of sub-cubes of side length equal to $11.0 \sigma$ is equal to 8 in the $x$ and $y$ direction, and 21 in the $z$ direction, which is the straining direction, see later. The structures are subsequently cut in half on a plane perpendicular to the $z$ normal direction, in the sense that we remove the bonds between adjacent beads along the z-axis and the two halves generated in this way are separated to allow the insertion of the spheres that mimic the nanoparticles, Figure 1(b). The spheres are added randomly on a 2-dimensional grid parallel to the just-created polymer surfaces. In this work we investigate a range of nanoparticle radii, $\mathrm{R}_{\mathrm{NP}}$ hereafter, from $1.0 \sigma$ to $7.0 \sigma$. Note that $\mathrm{R}_{\mathrm{NP}}=5.0 \sigma$ is a critical value as the diameter of these particles is equal to the mesh size. The insertion probability of a nanoparticle on a given vertex of the 2-dimensional 


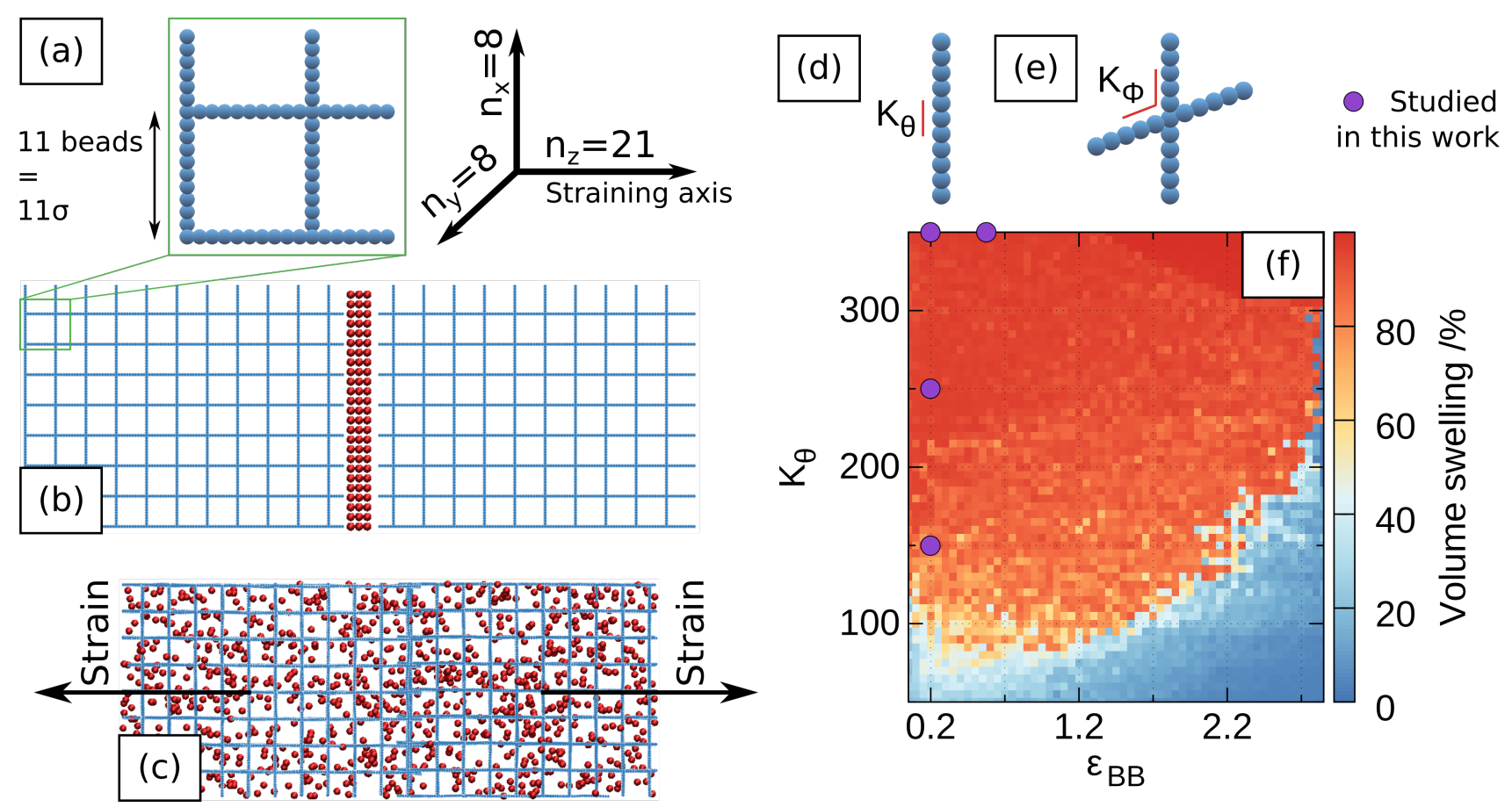

Figure 1: Polymer beads are colored in blue, while the nanoparticles are in red. (a) shows the base cube unit used, and the number of repetitions in each dimension. In (b) and (c) we show an example structure before and after equilibration, respectively. (d) and (e) sketch the two angular contributions to the potential energy of the system. Note that for any choice of $k_{\theta}, k_{\phi}=k_{\theta} / 2$. Finally, (f) shows the degree of swelling as a function of the angular stiffness and polymer-polymer interaction parameter, $\epsilon_{\mathrm{BB}}$. The purple dots correspond to the network configurations studied in this work.

grid is set to $65 \%$; this is done to allow for randomness in the distribution of placed particles. Once all the vertices on a given insertion plane have been visited, a new grid, displaced in $z$ from the first one, is created and the whole process repeated until the desired number of nanoparticles has been added to the structure. The total number of nanoparticles inserted is governed by a ratio defined as $\mathrm{A}_{\mathrm{P}}=N_{\mathrm{NP}} \pi \mathrm{R}_{\mathrm{NP}}^{2} / \mathrm{A}_{\text {Interface }} . N_{\mathrm{NP}}$ is the total number of inserted particles, and $\mathrm{A}_{\text {Interface }}=L_{x} L_{y}$ is the area of the interface (perpendicular to the straining direction $z$ ). Thus, $A_{P}$ measures the fraction of area, before equilibration, covered by the nanoparticles. In this study $A_{P}=0.4$ to ensure no double layer of nanoparticles is created. ${ }^{4}$ In fact, similarly to what we observed in our previous investigation on collapsed gels, ${ }^{4}$ a double layer also forms in the swollen gel for $A_{P} \approx 1$ and nanoparticles' radii greater than the mesh size. However, such double layer prevents adhesion, whereas here we are 
interested in the application-relevant regime where the nanoparticles act as a glue between the two interfaces. Additionally, structures with $A_{P}=0.0$, corresponding to the case of no inserted nanoparticle, are generated to be used as a benchmark. Finally, the structures are equilibrated broadly following the recipe from previous works, $\underline{410}$ and they are ready to undergo the straining procedure Figure 1(c). Briefly, the structures undergo a set of compression/decompression and heating/cooling cycles in search of a lower energy configuration. A complete description of the equilibration stages is provided in the supplementary information, Section 1. For every set of parameters, four structures are independently generated to be used to compute ensemble averages and uncertainties.

\section{Model}

As it is not our aim to study a specific polymer-nanoparticle system, Lennard-Jones reduced units are adopted throughout the paper unless stated otherwise. Hence, the mass $m$, and the parameters of the inter-particle interaction $\sigma$ and $\epsilon$ are set to unity. Rescaling by (an appropriate combination of) these parameters has the effect of making all other quantities

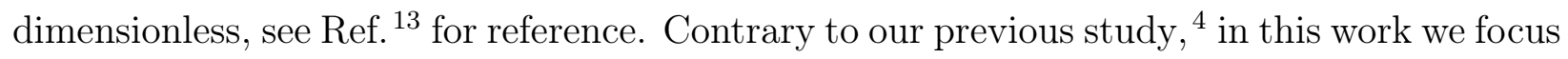
on the case of a polymer in good solvent conditions, i.e., the network is in a completely swollen state. For this reason, whereas we still use a bead-spring model, this is augmented with an angular term to allow to control the gel stiffness. As also previously shown in, 11 a crosslinked network described in this way exhibits a swollen-to-collapse transition that depends both on the non-bonded bead-bead interaction as well as on the parameter controlling the angular potential, see also Figure 1(f) for reference. This allows us to simulate different types of swollen gels, as discussed later.

In practice, as in, $\underline{411}$ the Hamiltonian of the system is split in bonded and non-bonded terms. The energy contribution of the bonded beads can be divided into two terms: bonding 
pairs and angular triplets, Equation 1.

$$
\begin{aligned}
U^{\text {Bonded }}(r) & =U^{\text {Bonds }}(r)+U^{\text {Angles }}(r) \\
& =\frac{k_{b}}{2}\left(r-r_{0}\right)^{2}+\frac{k_{\theta}}{2}\left(\theta-\theta_{0}\right)^{2}+\frac{k_{\phi}}{2}\left(\phi-\phi_{0}\right)^{2}
\end{aligned}
$$

The potential energy has an harmonic form for both angles and bonds, Equation 2. For the bond part, $k_{b}=80.0$ and $r_{0}=1.0$, and they are kept constant in this entire study. There exist two angular contributions to the potential energy Figure 1(d,e). The first, parametrized by the angle $\theta$, describes the edges of the polymer box, and $\theta_{0}=180.0$ while $k_{\theta}$ varies. The second, parametrized by the angle $\phi$, describes the vertices of the box, and its parameters are $\phi_{0}=90.0$ and $k_{\phi}=k_{\theta} / 2$. Figure 1(f) summarizes the choice of parameters for this study. We study gels: 1. close to the collapsing point (i.e., soft), 2. far away from the collapsing point (i.e., stiff), 3. in between 1. and 2., and 4. stiff with relatively strong polymer-polymer affinity. Note that every choice produces a swollen configuration, in contrast with our previous work focused on collapsed gels. $\stackrel{4}{4}$

The non-bonded contribution to the potential energy for the interaction between polymer beads as well as for the polymer beads-nanoparticle and nanoparticle-nanoparticles interactions is modeled by a truncated and (double-)shifted Lennard-Jones potential $U_{\text {TSLJ, }}$ Equation 3.

$$
U_{\mathrm{TSLJ}}^{i}(r)=\left\{\begin{array}{lr}
4 \alpha_{i}\left[\left(\frac{\sigma}{r-\Delta_{i}}\right)^{12}-\left(\frac{\sigma}{r-\Delta_{i}}\right)^{6}\right]-U_{\mathrm{LJ}}\left(r_{i}^{\mathrm{c}}\right) & \Delta_{i}<r \leq r_{i}^{\mathrm{c}}+\Delta_{i}, \\
0 & r>r_{i}^{\mathrm{c}}+\Delta_{i}, \\
+\infty & 0<r \leq \Delta_{i} .
\end{array}\right.
$$

Where $U_{\mathrm{LJ}}$ corresponds to a simple 12-6 Lennard-Jones potential. $i$ represents the type of non-bonded interaction $i \in$ [Bead-Bead, Bead-Nanoparticle, Nanoparticle-Nanoparticle]. Each interaction has its own set of parameters $\alpha_{i}, \Delta_{i}$, and $r_{i}^{c}$, and all are summarized 
in Table 1. $\alpha_{i}$ controls the strength of the interaction. In this study, different values for

Table 1: Summary of all the potential parameters used in this work for the description of the non-bonded interactions of the system. The meaning of $\alpha_{i}$, $\Delta_{i}$, and $r_{i}^{c}$ can be found in the main text. $\mathbf{R}_{\mathrm{NP}}$ is the radius of the inserted nanoparticles.

\begin{tabular}{cccc}
\hline$i$ & $\alpha_{i} / \epsilon$ & $\Delta_{i}$ & $r_{i}^{c} / \sigma$ \\
\hline Bead-Bead (BB) & Varies & 0.0 & $2 \sqrt[6]{2}$ \\
Bead-Nanoparticle (BN) & Varies & $\mathrm{R}_{\mathrm{NP}}-\sigma / 2$ & $2 \sqrt[6]{2}$ \\
Nanoparticle-Nanoparticle (NN) & 1.0 & $2 \mathrm{R}_{\mathrm{NP}}-\sigma$ & $\sqrt[6]{2}$ \\
\hline
\end{tabular}

$\alpha_{\mathrm{BN}} \equiv \epsilon_{\mathrm{BN}}$, corresponding to the polymer bead-nanoparticle strength of interaction, also sometimes referred to as (chemical) affinity, are investigated. This is done to mimic the effect of different nanoparticles surface chemistry, which can be used to control the attraction with the polymer gel. ${ }^{4} \Delta_{i}$ shifts the interaction to account for excluded volume effects. Its value is chosen so that $\Delta_{i}=R_{1}+R_{2}-\sigma$, where $R_{1}$ and $R_{2}$ are the radii of the two particles taking part to the interaction. ${ }^{4|10| 14}$ In other words, this choice shifts the zero of the $U_{\text {TSLJ }}$ to a distance equal to the sum of the two radii. Finally, $r_{i}^{c}$ corresponds to the distance at which the potential is truncated and shifted so that the force and energy are zero. Experimentally, nanoparticles for gluing hydrogels must have minimal interaction among each other. This is a necessary requirement for practical applications because if a sizeable attraction was present, the solution would not be stable and nanoparticles would quickly coagulate over time. Consequently, as in previous studies, ${ }^{4 \mid 10[14}$ we mimic this condition by truncating and shifting their interaction at the minimum of the Lennard-Jones potential, resulting in a purely-repulsive interaction equivalent to a WCA potential. ${ }^{15}$ We note that this paper focuses on the effect of the nanoparticles' size and effective nanoparticle-polymer bead interaction. The rich questions associated with more complex nanoparticle-polymer interaction as well as nanoparticles' shape $\mathrm{e}^{16}$ is the subject of ongoing investigations and will be published in a future work. 
All the coarse-grain molecular dynamics simulations are performed using the Large-scale Atomic/Molecular Massively Parallel Simulator (LAMMPS) package. ${ }^{17}$ A velocity-Verlet integrator with a timestep of $\delta t=10^{-4}$ is used to evolve the equations of motion. A Nosé-Hoover thermostat and barostat is adopted to control the temperature and pressure, respectively. .28 The pressure (in reduced units) is set to $P=0.0$, and the temperature to $T=0.8$. All the presented snapshots of the structures are rendered using $\mathrm{VMD}^{21}$ with the TopoTools plug-in.

\section{Uniaxial Straining}

Once equilibrated, the structures are uniaxially strained along the $z$-axis within the $N T L_{z} \sigma_{x x} \sigma_{y y}$ ensemble, as in previous studies. $\frac{4 \mid 1022}{10}$ In this ensemble the total number of particles, $N$, and temperature, $T$, are maintained constant, and the stresses normal to the straining direction, $\sigma_{x x}$ and $\sigma_{y y}$, are kept equal to zero. During the straining procedure the length of the simulation box along the $z$-axis, $L_{z}$, is increased at a uniform strain rate, $\dot{\eta}$, such that $L_{z}(t)=L_{z}(0)(1+\dot{\eta} t)$. The choice of $\sigma_{x x}=\sigma_{y y}=0$, i.e., zero lateral stresses, is done to mimic the usual boundary conditions on an experimental tensile test at a constant

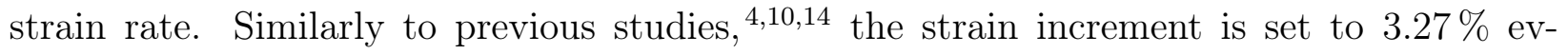
ery $\tau=\delta t^{-1}=10^{4}$ timesteps, in other words, the simulation box is strained along $z$ by $0.000327 \%$ every timestep. This strain rate is chosen because at this value the stress-strain curve does not strongly depend on the strain rate, or, in other words, our straining procedure is equivalent to straining under quasi-equilibrium conditions. The straining is performed for $100 \tau$, hence the total strain on the system at the end of the procedure is $327 \%$. The stress along the straining direction $\sigma_{z z}$ is given by the negative of the $z z$-component of the pressure tensor $P_{z z}$. For every combination of parameters, the reported stress-strain curve is the averaged result over four independently-generated structures. The width of the shading osculating the stress-strain curves signifies $\pm 1 \sigma_{s}$, where $\sigma_{s}$ is the standard deviation of the 
calculated stress over the ensemble of structures.

\section{Results and Discussion}

We first perform a large set of simulations in the NPT ensemble spanning various combination of $\epsilon_{\mathrm{BB}}, k_{\theta}$ (the former is the polymer bead-polymer bead interaction strength, the latter the angular stiffness) in order to extract the swelling diagram of the gel, as presented

in Figure 1(f). We then choose four representative points, all within the swollen region so as to obtain a model representative of an hydrogel under hydrated conditions. From there, we begin by studying the nanoparticles' potency to glue together the interface between two polymer networks as a function of the the polymer bead-nanoparticle affinity, as measured by $\epsilon_{\mathrm{BN}}$, and the nanoparticles' radius. With the goal to provide a more transferable link between our work and experimental efforts, contrary to our previous study, ${ }^{4}$ we present our reinforcement results in terms of adhesion strength relative to the unfilled interface. The adhesion energy is calculated as the integral of the stress-strain curve, where the integration extrema are taken to be the first two times the curve crosses the zero-stress axis, see Figure 2 for reference. The relative adhesion strength, defined as the percent change in adhesion energy between the two half of the gel when comparing a system with and without nanoparticles, is reported in Figure 3. The color-code is so that, going from blue to red, the adhesion between the two polymeric interfaces is weakened to strengthened, respectively, with white showing a no-change scenario with respect to the unfilled case where no particles are present at the interface. In (a), (b) and (c) we study polymer networks with different rigidity, $k_{\theta}=150$ and $\epsilon_{\mathrm{BB}}=0.2, k_{\theta}=250$ and $\epsilon_{\mathrm{BB}}=0.2$ and $k_{\theta}=350$ and $\epsilon_{\mathrm{BB}}=0.2$, respectively. In (d) we show the results for a network as rigid as in (c), but with stronger polymer bead-polymer-bead interaction, $k_{\theta}=350$ and $\epsilon_{\mathrm{BB}}=0.5$. These combinations correspond to the purple points marked in the gel phase diagram presented in Figure 1(f) and correspond to an hydrogel that is always in the swollen state, albeit with different physical 


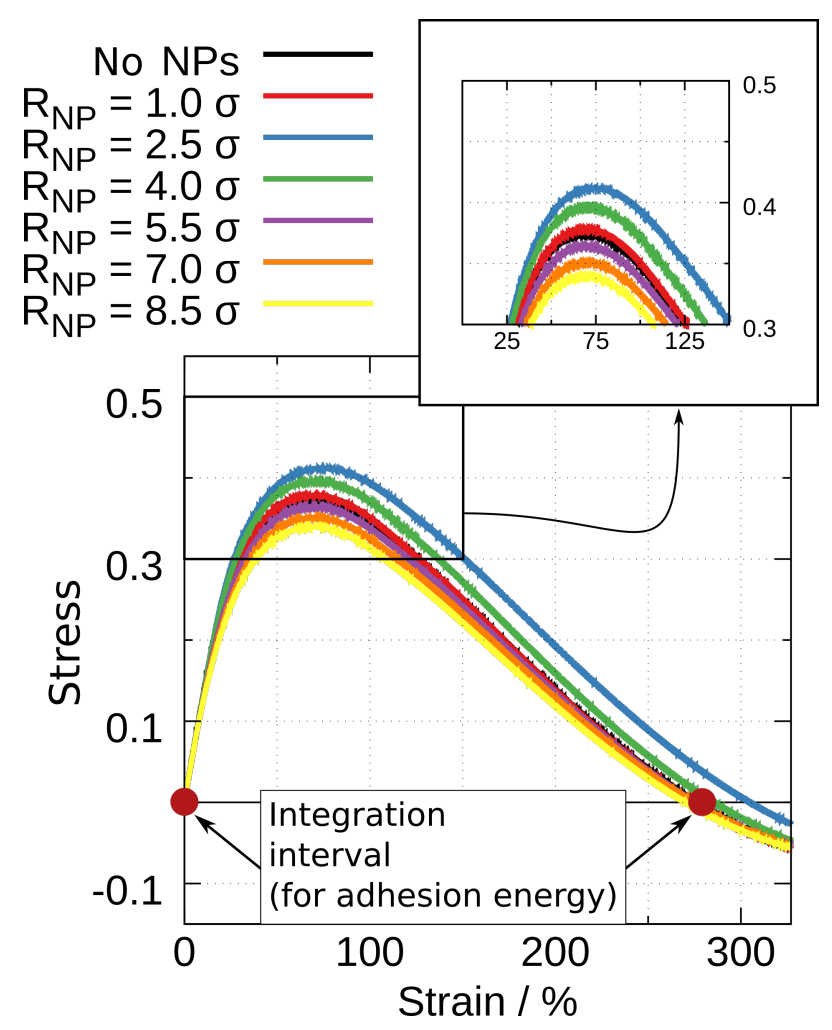

Figure 2: Example stress-strain response for different $\mathrm{R}_{\mathrm{NP}}$ at constant $\epsilon_{\mathrm{BN}}=1.6 \epsilon$, and network $k_{\theta}=350$ and $\epsilon_{\mathrm{BB}}=0.5$. The adhesion energy is here defined as the integral between the two zero of each stress-strain curve. While not visible, the ensemble uncertainty is represented as shaded area around the mean curve.

properties.

We make the following general observations. First, regardless of the combination of $\left(k_{\theta}, \epsilon_{\mathrm{BB}}\right)$ chosen to obtain a swollen gel, the qualitative trends in adhesion are all very similar. In other words, there is a robust reinforcement behaviour as a function of network/strand stiffness and polymer-polymer affinity (a proxy for the polymer surface tension). Second, not all combinations in terms of nanoparticle bead-polymer affinity and nanoparticle size provide interface reinforcement. In fact, for a large part of the parameter space spanned, the interface becomes less stable in the presence of nanoparticles. To facilitate the discussion of trends, and because this turns out to be the relevant microscopic length-scale to rationalise the behaviour of the system, we introduce the scaled size of the nanoparticles $\widetilde{R}$, a non-dimensional 


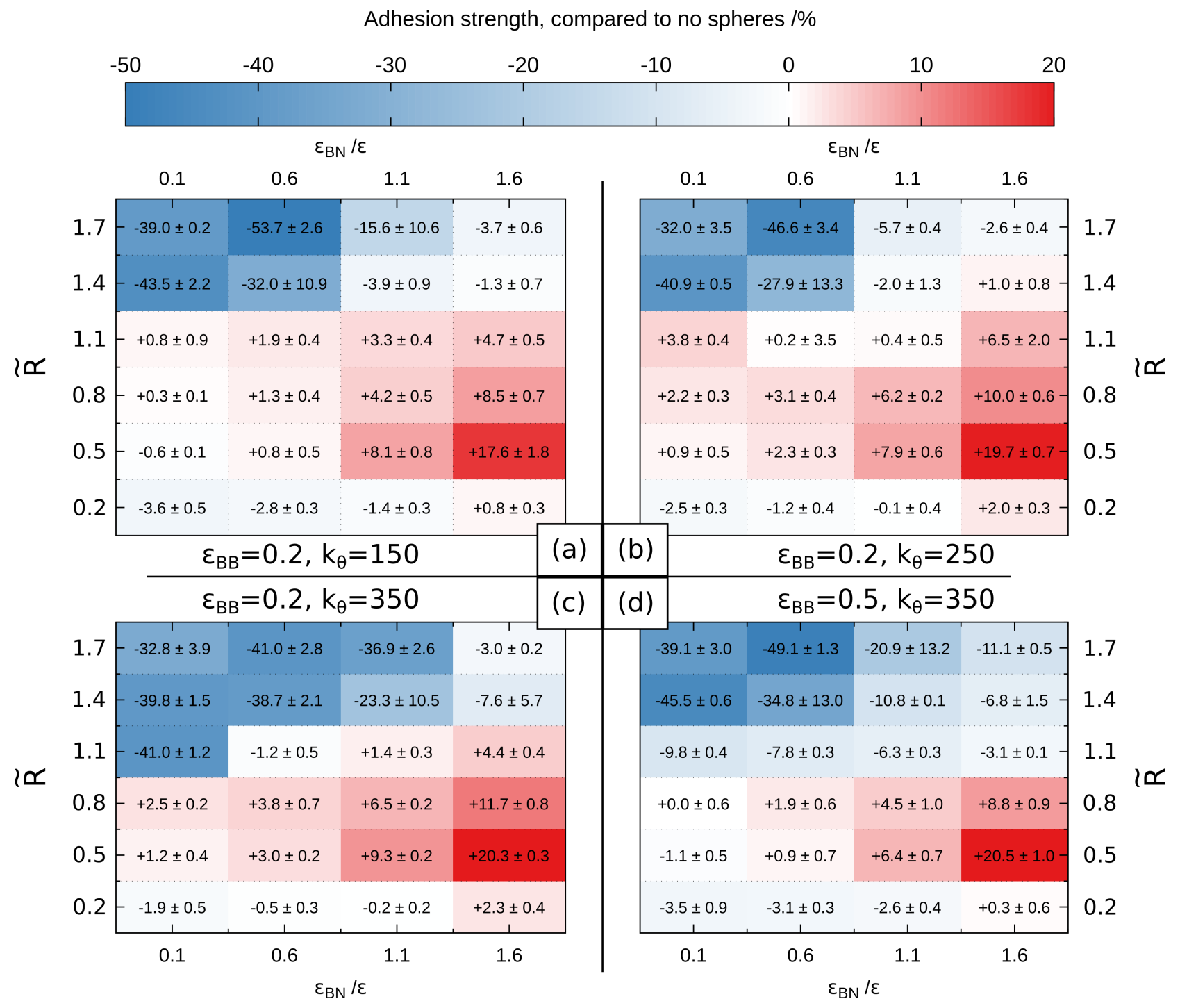

Figure 3: Summary of the adhesion energy as a percentage change with respect to the no nanoparticles case.

quantity defined as $\widetilde{R}=2 R_{\mathrm{NP}} / L_{m}$, i.e., the ratio between the diameter of the nanoparticles and the mesh size. We remind the reader that $L_{m}$ is kept fixed at $L_{m}=10 \sigma$ throughout this study. For a fixed nanoparticles' size, adhesion typically increases with increasing polymer bead-nanoparticle affinity, as one would probably expect given that stronger "bonds" are formed that can sustain more stress and thus help keep the gel-gel interface together during deformation. An exception to this rule is the highest nanoparticle size $(\widetilde{R} \gg 1)$, for which a non-monotonic behaviour is observed. However, its importance for applications is limited, for the simple reason that it occurs in a region of the parameters space where the 
presence of nanoparticles either marginally reinforce the interface, or makes it even weaker. In other words, this exception occurs where nanoparticles do not behave as a functional glue. Instead, an application-relevant non-monotonic trend is observed when one looks at adhesion vs nanoparticle size. This non-monotonicity, as we will explain later, results from the coupling of different physical parameters. Generally speaking, we observe that in this case the interfacial stability increases for decreasing nanoparticles' size up to an optimal value in the interval $0.5<\widetilde{R}<0.8$, i.e., slightly below the mesh size, and then decreases again for smaller nanoparticles up to a point where their effect is completely negligible.

Understanding the origin of the different behaviours and their dependence on the system's parameters requires looking at the detailed microscopic picture provided by the simulations. Crucially, this is also important to tell us whether what we observe is relevant for real systems, or just a consequence of the limitations and assumptions of our coarse-grained model. As a first microscopic characterization of the system, we thus investigate the spatial distribution of the nanoparticles, as well as the spatial density of the polymer, after the initial equilibration, but before the straining procedure occurs. For this analysis, at a given snapshot the structure is sliced in planes spanning the whole $x$ and $y$ directions, and of thickness equal to $2 \sigma$ along the $z$ direction; the numbers of polymer beads and nanoparticles in a given slice is then counted. The total number of analysed snapshots per combination of parameters is 1000 , separated in time by $0.05 \tau$ to decrease their temporal correlation. The final reported quantity is the (normalised) number density for both polymer beads and nanoparticles, Figure 4. In analysing these results, we remind the reader that nanoparticles are initially inserted only at the interface between the two gels. From (a) to (d), the scaled radius of the nanoparticles increases from $\widetilde{R}=0.2$ to $\widetilde{R}=1.4$. These results are reported for a polymer network characterised by $k_{\theta}=350$ and $\epsilon_{\mathrm{BB}}=0.5$ but they are representative in general for all polymer networks we have studied in the swollen state. The insets show snapshots from the molecular dynamics simulations used to compute the polymer beads and nanoparticles number density distributions. As expected based on its periodicity and sym- 


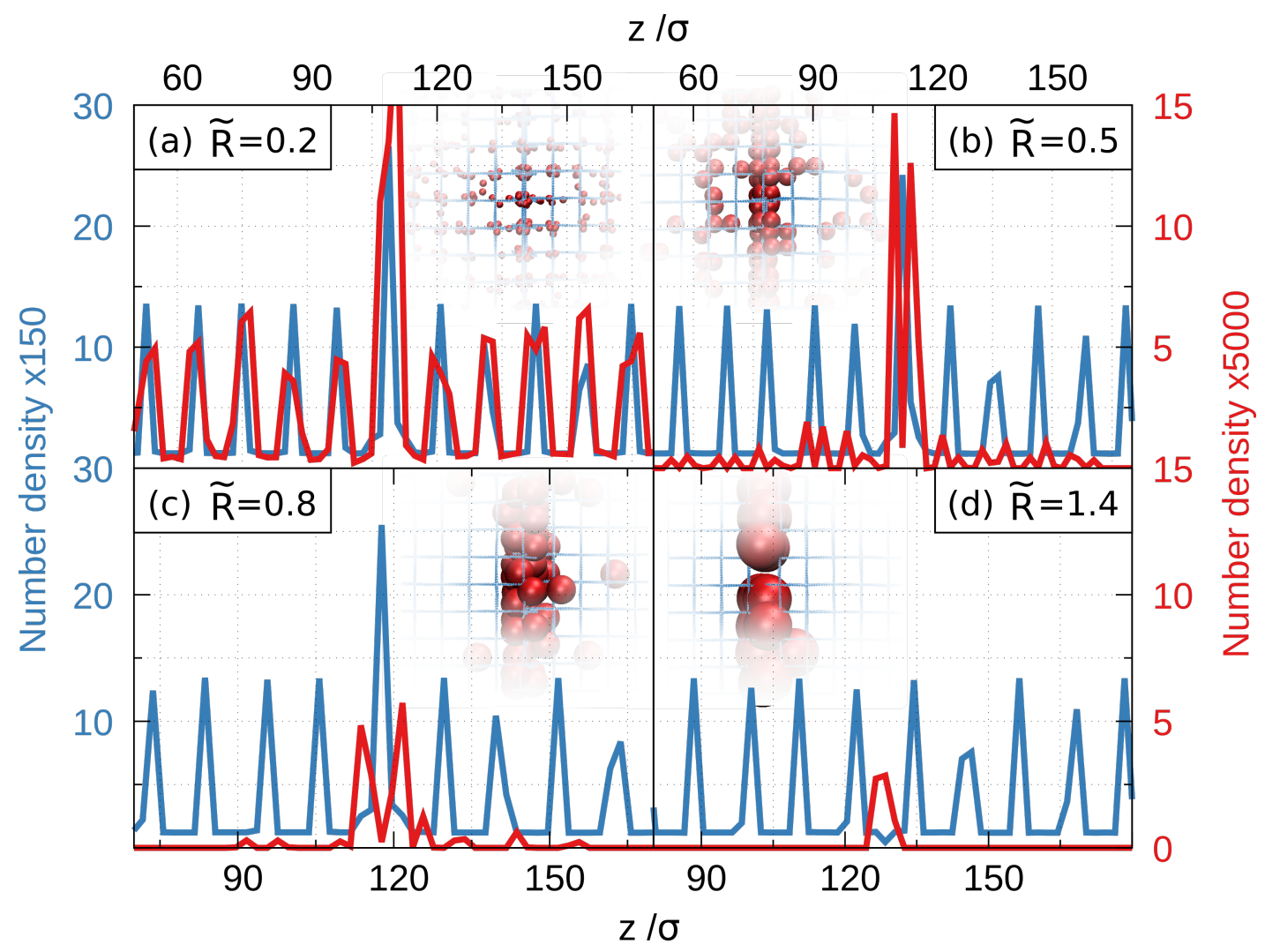

Figure 4: Polymer beads (blue) and nanoparticles (red) spatial distribution. All plots refer to a network with $k_{\theta}=350$ and $\epsilon_{\mathrm{BB}}=0.5$. From (a) to (d), $\widetilde{R}$ is increased from $\widetilde{R}=0.2$ to $\widetilde{R}=1.4$.

metry, the polymer density shows sharp, regularly-spaced by $\sim 11 \sigma$ peaks, corresponding to the edges of the polymer network cages. Notably, for $\widetilde{R}<1$ and thus nanoparticles smaller than the polymer network mesh size (see Figure 1(a-b) for reference), the two interfaces of the polymer network are in direct contact with each other, and the network partially interpenetrate for a distance of $\approx L_{m}$ hence the increase (in fact, the doubling) in the local density of polymer in this interface region. This is not possible for bigger radii, where the nanoparticles act as spacing agents that bridge the two interfaces, see Figure 4(d), and in fact in this case the polymer density at the interface is depleted compared to the bulk. Regarding the nanoparticles spatial distribution, we observe that the small nanoparticles are not confined at the interface $(a)$ and diffuse throughout the whole system during the equilibration procedure. Instead, the more comparable $R_{N P}$ is to the mesh size, the more 
the nanoparticles concentrate at the interface between the two polymer networks instead of spreading, and for nanoparticles equal to the mesh size or larger, $(c)$, they remain confined exclusively at the interface. This density distribution provides a simple picture to explain the monotonic behaviour in adhesion observed. For particles to act as bridges between the two polymer networks, they must both i) reside at the the interface between them and ii) form the highest possible number of contacts with them. When particles are too small, they simply diffuse away, violating criteria i), whereas when particles are too large, their presence lowers the amount of polymer at the interface, and thus the number of possible bonds that can be formed (the latter point is also more directly visible from the results of Figure 7 , which will be discussed later). As a result, there is an optimal particle size at which adhesion should be maximised, hence the non-monotonic behaviour observed in simulations.

Although the initial nanoparticle profile well explains the observed behaviour, it also brings the important question of how relevant are the simulation results to practical applications. This is because, assuming equilibrium is reached before the straining procedure, the spatial density of nanoparticles will strongly depend on the simulated system size. For our system, and, in general, for any system size accessible via molecular simulations, this is far from being representative of reality because the space occupied by the interface with respect to the bulk is greatly exaggerated. We can make a simple order-of-magnitude calculation to explain this point. Imagine we need to glue together two pieces of skin of thickness $L_{s}=1 \mathrm{~mm}$, using nanoparticles of the order of the extra cellular matrix mesh size. As an approximation, we take the mesh size to be $L_{m}=100 \mathrm{~nm}$, which also sets the order of magnitude of the interface, since this is approximately the size of the region where confined nanoparticles will be able to act as bridges. The bulk to surface ratio is thus $L_{s} / L_{m}=\mathcal{O}\left(10^{7}\right)$ in this realistic setting, compared to $\mathcal{O}\left(10^{1}\right)$ in our simulations. In practice, this means simulations underestimate the probability to be in the bulk rather than at the interface by a factor of $10^{6}$. Thus, if particles can diffuse within our simulation timescale and reach equi- 
librium, we would expect their concentration at the interface, and any effect in terms of changing the adhesion energy, to be over-estimated. For the smallest particles we have simulated, where the reinforcement is already almost statistically irrelevant, this means it will probably be zero in the real system, simply because they will readily diffuse even more into the bulk, leaving a vanishingly small concentration at the interface. But what about larger sizes? More precisely, is their reinforcement purely due to the limitations in time-scales of molecular simulations, i.e., the system not yet having relaxed to equilibrium within the simulation timescale? If so, are the results on reinforcement at all relevant for real systems and time-scales?

To answer this question, we need to understand something more about the kinetics of the system, in other words, it is crucial to characterise the diffusion time-scale of the nanoparticles. We do this by first computing the diffusion profiles as a function of nanoparticle size and affinity with the polymer, with the idea to obtain some insight to extrapolate the nanoparticles' behaviour in the limit of greater time- and length-scales. To do so, after the initial equilibration time (which, as we have seen, might only be enough to reach local equilibrium) the system is evolved for $1.2 \mathrm{G} \delta \mathrm{t}=120 \mathrm{k} \tau$ in the canonical ensemble. The volume is kept equal to that of the last stage of the equilibration routine (thus before straining), and the temperature is maintained constant at $T=0.8$. Throughout the simulation the position vector of every nanoparticle in a given structure is recorded every $5 \tau$ for postprocessing. This approach and simulation setup is similar to what has been used by Kim et $a l . \stackrel{23}{23}$ for studying the diffusion of particles within a polymeric membrane, with the difference that in our case the local polymer density, see Figure 4, is not periodic but instead exhibits a maximum for $2 R_{N P}<L_{m}$ and a minimum for $2 R_{N P} \geq L_{m}$. The mean-squared displacement (or MSD, defined as in Equation 4) is used to extract the self-diffusion constant of the 
nanoparticles.

$$
\begin{aligned}
\mathrm{MSD}_{i} & =\left\langle r^{2}(t)\right\rangle_{i}=\left\langle|\vec{r}(t)-\vec{r}(0)|^{2}\right\rangle_{i} \\
D_{i} & =\frac{1}{6} \lim _{t \rightarrow \infty} \frac{\partial \operatorname{MSD}_{i}(t)}{\partial t}
\end{aligned}
$$

Where $i$ indicates the species index, $\vec{r}$ the position vector, $\langle\cdot\rangle_{i}$ the ensemble average, and, finally, $\mathrm{MSD}_{i}$ is the MSD of species $i$. Figure 5 shows example drifts of the MSD as a function of time for different set of parameters. Figure 5(a) shows the MSD dependence on

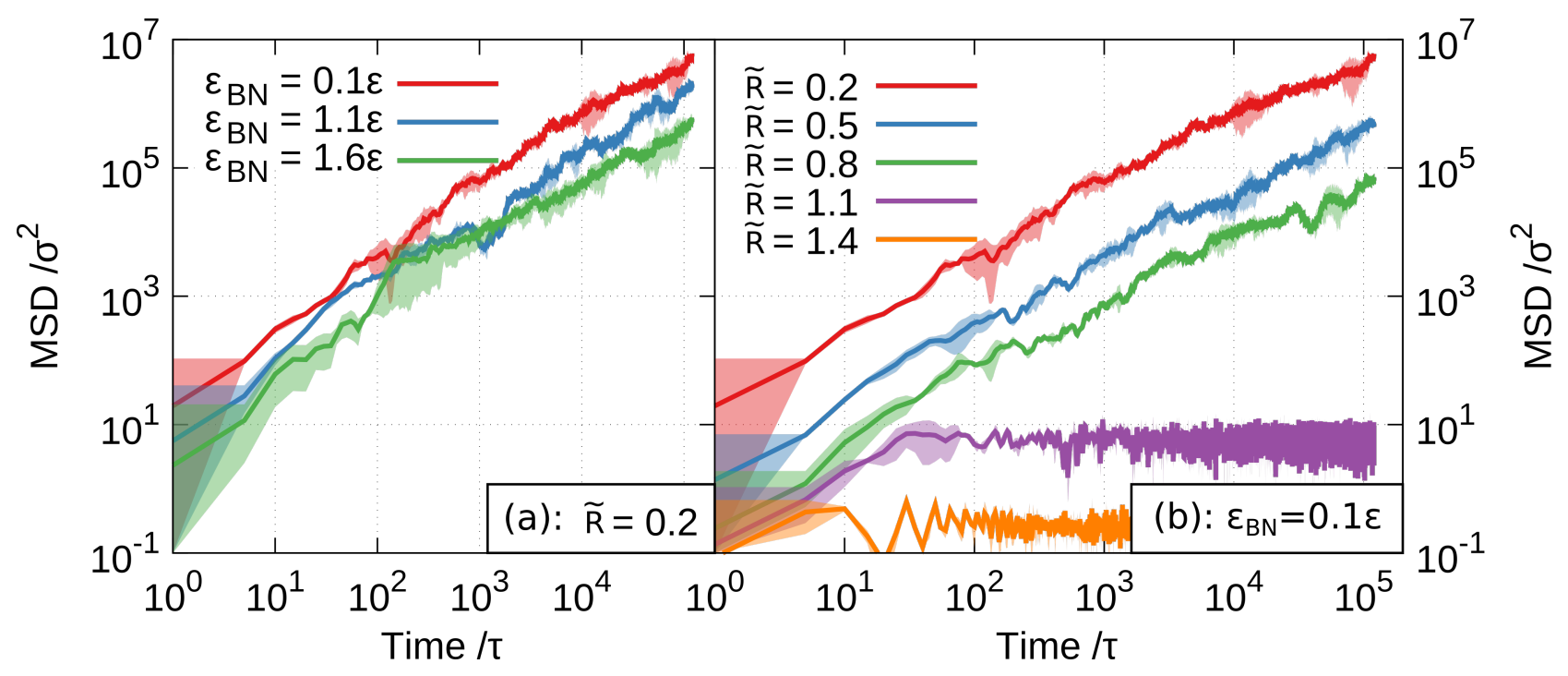

Figure 5: Mean-squared displacement for (a) different polymer bead-nanoparticle interactions, and (b) different $\widetilde{R}$. All results are for a network with $k_{\theta}=350$ and $\epsilon_{\mathrm{BB}}=0.5$.

the nanoparticle-polymer interaction parameter for a fixed $\mathrm{R}_{\mathrm{NP}}=1.0 \sigma$. Because, similarly to, ${ }^{23}$ we are studying the diffusion of a particle within a polymer network and we work at relatively low packing fractions, we expect that a stronger $\epsilon_{\mathrm{BN}}$ would exponentially slow the nanoparticle's drift (equivalent to a rigid downward vertical shift in the log-plot), an hypothesis that is borne out by our computational experiment. This exponential dependence on epsilon is what one would expect for a system that moves via a hopping mechanism, a result that we will exploit later. The other important factor to consider is the MSD drift dependence on the nanoparticles' radius shown in Figure 5(b). From $\widetilde{R}=0.2$ to $\widetilde{R}=0.8$, 
the MSD shows the characteristic monotonically-increasing linear dependence as a function of time, albeit larger particles drift slower than smaller ones. However, for $\widetilde{R}=1.1$ the nanoparticles are larger than the polymer mesh size and do not diffuse at all, as indicated by the flat MSD over time, with an upper bound which is indeed around the polymer mesh-size $L_{m}$, signalling that large particles can only fluctuate within the cage formed by the polymer but never get out of it. This is reflected in Figure 5(b), where it is shown that the diffusion coefficient is suppressed for increasing $\widetilde{R}$, going to zero (within the statistical uncertainty) for $\widetilde{R}>1$.

Using Einstein's relation, $\frac{1324}{}$ the effective diffusion constant for species $i, D_{i}$, is computed from the slope of the MSD, Equation 5 . Figure 6 summarizes (a) the general trend in diffusion coefficient for $\epsilon_{\mathrm{BN}}$ and $\widetilde{R}$, and (b) an extended analysis of the diffusion coefficient versus $\widetilde{R}$ at fixed $\epsilon_{\mathrm{BN}}=0.1$. The behaviour for large particles in our system can be easily rationalised. Because we are working with a swollen, and thus relatively stiff and underformable polymer network, particles larger than the mesh size need to overcome an energy barrier to diffuse out of the interface where they are initially placed. This barrier comes from the fact that diffusion would require stretching the polymer network until it forms a hole larger than the particle, from which it can escape to a neighbouring site. Just to make an rough estimate, we can assume homogeneous stretching of the bonds in the polymer, which lead to an estimate for the energy barrier $\Delta E$ to scale as $\Delta E \approx k_{h}\left(l_{0}-\frac{\left(2 R-L_{m}\right)}{l_{0}}\right)^{2}$. Even for a particle only $10 \%$ larger than the mesh size, this would require tens of $k_{\mathrm{B}} T$, that is, energies comparable to those necessary for breaking an irreversible covalent bond. Based on these observations, for particles for which $\widetilde{R}>1$, we can expect particles to be confined at the interface not only in the timescale of our simulations, but also in real experiments, as indeed observed. ${ }^{8}$ In this case, we would expect simulation to be able to capture experiments. Compared to the case of larger particles, for particles smaller than the mesh size $(\widetilde{R}<1)$ no stretching of the network is needed for diffusion and thus there should be no steric barrier to confine them at 


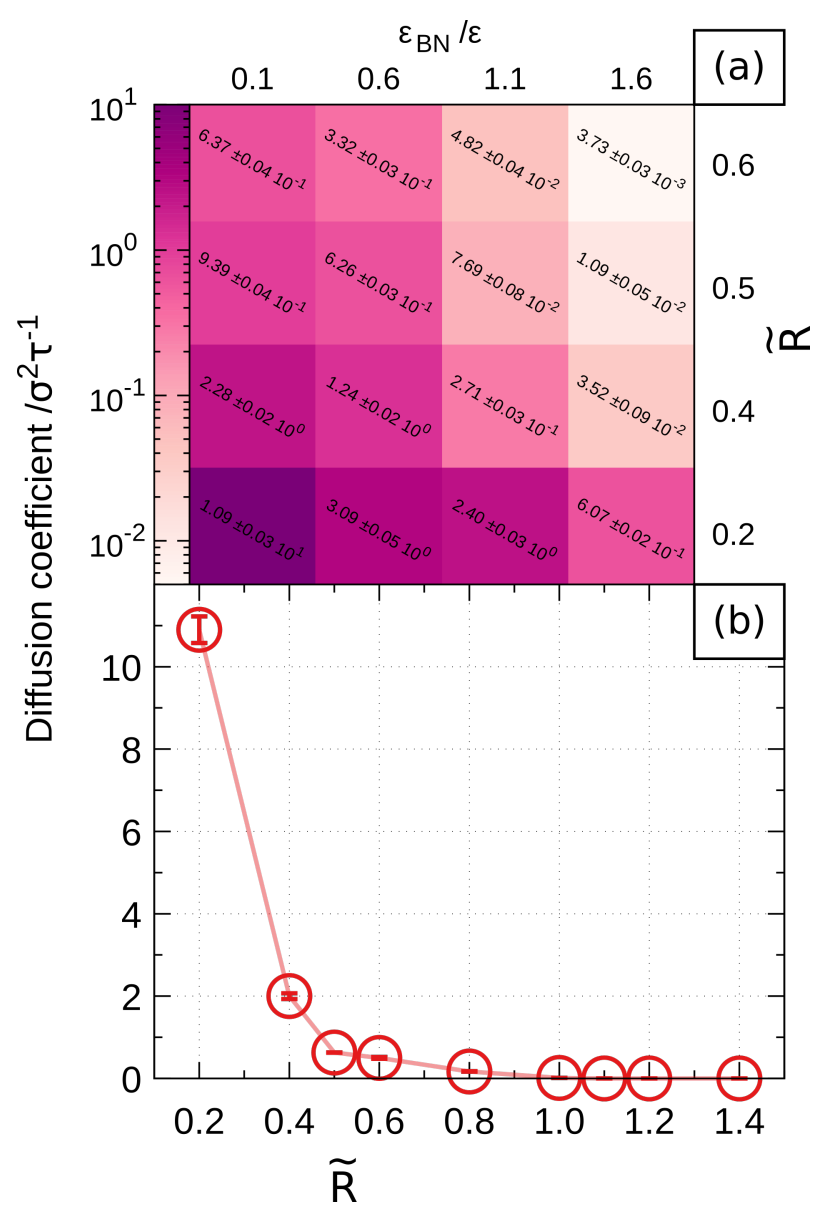

Figure 6: Diffusion coefficient analysis for a network with $k_{\theta}=350$ and $\epsilon_{\mathrm{BB}}=0.5$.

the interface. In this case, particles should be able to quickly reach their equilibrium density profile, which, as we previously argued, will strongly differ in real-size systems compared to those that can possibly be simulated. Although particles in this size regime do not need to stretch the polymer network to diffuse, they still need to hop out an energy well in order to reach their equilibrium state, a well due to the interaction of the polymer with the nanoparticle. One should expect an energy minimum at the interface because, as shown in Figure 4 and previously discussed, for these smaller nanoparticles the surfaces of the two gels can get in direct contact with each other. The higher local density of polymer obtained in this way increases the local number of attractive polymer bead-nanoparticle interactions compared to the bulk. In fact, this hypothesis is confirmed in Figure 7, where we show the polymer bead-nanoparticle interaction energy difference between the interface 
and the bulk calculated from our simulations, for varying nanoparticles-polymer affinities and particles' sizes. As it is clearly visible, for all the investigated parameters the interaction

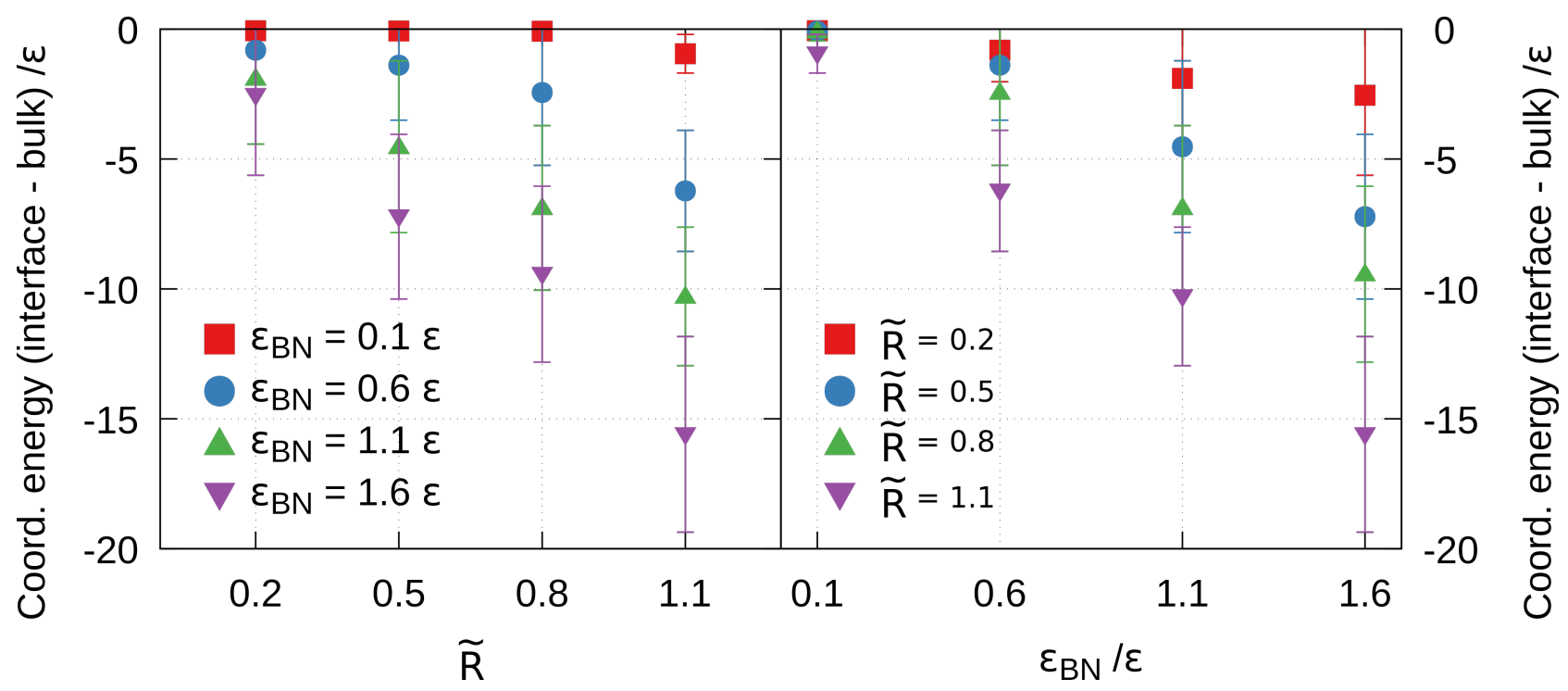

Figure 7: Particle-network interaction energy difference (interface - bulk) as a function of the particle radius, left, and polymer bead-nanoparticle affinity (right). For all set of parameters, the energy of the particle at the interface is always lower than in the bulk, providing a kinetic trap able to confine them for long time-scales (see main text).

energy difference is negative, i.e., there is a stronger polymer-nanoparticle interaction at the interface compared to the bulk. In practical terms, the presence of this energy minimum means that particles are kinetically trapped at the interface on timescales $t_{e}$ less than a critical escape time $\tau$ or in other words the system can only reach its thermodynamic equilibrium state for $t_{e}>\tau$. However, as we have previously explained, our simulations (or, more precisely, any computationally tractable simulation aiming to describe nanoscale features) necessarily overestimates the equilibrium density of nanoparticles at the interface. Thus, the microscopic picture arising from these simulations for particles that could diffuse in the bulk is only correct for experiments and applications where the relevant timescale is smaller than $\tau$. An estimate is easily given using Kramer's theory of escape for brownian particles in an energy well,, 25 which gives: 


$$
\begin{aligned}
\tau & =\tau_{0} \exp (-\beta \Delta E) \\
& =\frac{L_{m}^{2}}{D_{\text {bare }}} \exp (-\beta \Delta E) \\
& =\beta L_{m}^{2} 6 \eta \pi R_{\mathrm{NP}} \exp (-\beta \Delta E)
\end{aligned}
$$

where $\Delta E$ is the depth of the energy well, $\beta=1 / k_{B} T$ being the inverse thermal energy, with $k_{\mathrm{B}}$ being Boltzman's constant and $T$ temperature. In deriving Equation 6, we have assumed that the well has about the same size of the polymer mesh $L_{m}$, meaning that once nanoparticles can move for such a distance they are free to escape to the bulk. $\eta$ is the viscosity in the polymer gel and we have used Einstein's relation to approximate the diffusion coefficient of the nanoparticle. Let us now consider nanoparticles with a size close to that of the polymer mesh, $\widetilde{R} \approx 1$. Using the viscosity of water as a lower bound to the real viscosity in a hydrogel, and considering a gel with an approximate mesh size of $L_{m}=100 \mathrm{~nm}$, corresponding to that of an archetypal biogels such as collagen,, Equation 6 predicts that a localisation energy of $\Delta E \approx 21 k_{B} T$, would keep a nanoparticle at the interface for a time of around one day. Because $\epsilon=5 / 4 k_{B} T$ in our units, or in other words $\Delta E \approx 17 \epsilon$, the required energy well we just calculated is actually twice deeper than what we obtain for our choice of parameters in the simulations, see Figure 7. Considering that $\Delta E$ scales linearly with affinity $\epsilon$ (see Figure 7, right panel), in experiments one would need an affinity around a factor of two larger than what we use here to see any appreciable interface reinforcement for $\widetilde{R} \approx 1$. At this affinities, however, the microscopic picture arising from nanoparticles confinement described by simulations would also hold, and we would expect to observe the non-monotonic behaviour of reinforcement as a function of nanoparticle size that we described.

Concerning the initial question we wanted to address, whether or not there is an optimal nanoparticle size for reinforcement, our results can be thus summarised in the following 
way: For very small sizes $(\widetilde{R} \ll 1)$, nanoparticles readily diffuse in the bulk and the fraction retained at the interface will be virtually zero in the relevant limit of nearly-infinite bulk-to-interface ratio. Because in the bulk they will not contribute to binding, they will not have any effect compared to a bulk system without nanoparticles, which is indeed what we observe. On the opposite side of the spectrum, nanoparticles' sizes above the mesh size $(\widetilde{R}>1)$ are trapped at the interface. However, because of their excluded volume, they generate a depletion of polymer at the interface (Figure 4). The lack of polymer co-localised with the particles reduces the number of bonds that can be done to bridge the two polymer networks. Furthermore, no network-network penetration occurs, further weakening the interface. Hence large nanoparticles also do not possess any bridging power, and in fact weaken the interface. Because of these two limits, the optimal size for reinforcement should thus be of the order of the mesh size $L_{m}$, neither much smaller, nor much larger, as indeed we observe. This size allows an increase in the local polymer density at the interface that has a twofold scope. On the one side, higher polymer density means a larger number of bridges between the two interfaces, hence more interface reinforcement. At the same time, this increase in density also increases the average polymer-nanoparticle (attractive) interaction at the interface compared to the bulk, which in turn allows to kinetically trap the nanoparticles where they are more useful. In the past, such optimal size was speculated, without however providing neither a systematic experimental data, nor any hint towards the reasons for such value. Crucially, we show that a strong non-monotonic behaviour with a maximum at $\widetilde{R} \approx 1$ should only be expected in experiments probing application-relevant timescales when high enough polymer bead-nanoparticle affinities are used, and no observable reinforcement should be expected otherwise, regardless of the nanoparticle size.

Before we conclude, we would like to discuss how to interpret the present results given another implicit approximation in our coarse-grained model. In our system, we study an ideal network, where all pores in the network have exactly the same size. We do this here 
to have a simple, more controllable system that can be used as a reference, and study the strengthening mechanism here before we move further up in complexity. In fact, in realistic biological hydrogels the polymer network will likely present a certain distribution of pore sizes (see e.g. $\frac{26127}{2}$ ). Our results suggest that polydispersity in the nanoparticles' size should be avoided or minimised. The presence of an optimal size of order $2 R_{\mathrm{NP}} \approx L_{m}$ (i.e., $\widetilde{R} \approx 1$ ) suggest that one should use nanoparticles with a size distribution as narrow as possible and in any case to avoid particles much larger than the mesh size. This is because only particles roughly around the mesh can strengthen the interface. Larger ones instead will weaken the interface and should thus be avoided, whereas much smaller ones, although not worsening the performance, will not contribute to strengthening because they will readily diffuse in the bulk. In fact, given that reinforcement is observed for the interval $0.5<\widetilde{R}<1.1$, a simple measurement of the efficiency of adhesion can be roughly approximated as:

$$
E=\int P(\widetilde{R}) f(\widetilde{R}) d \widetilde{R}
$$

, where

$$
f=\left\{\begin{array}{lll}
1 & \text { for } & 0.5<\widetilde{R}<1.1 \\
0 & \text { for } & \widetilde{R}<0.5 \\
-1 & \text { for } & \widetilde{R}>1.1
\end{array},\right.
$$

where the -1 contribution is introduced to penalise particles larger than the threshold value, which tend to decrease adhesion. Details of the approximations implicit in using ?? and ??, and of their range of applicability, can be found in the Supplementary Information. In practice, achieving a narrow size-distribution simply from their synthesis could be difficult but in this case separation techniques to filter nanoparticles based on their size should be used. This fact should also be taken into account in interpreting experiments aimed at measuring the nanoparticle gluing power, because chemically identical batches might lead to completely different results due to a different size distribution, leading to conflicting results. 


\section{Conclusions}

In this work, we explore the potency of spherical nanoparticles to mechanically reinforce the interface between two swollen polymers, using coarse-grained molecular dynamics simulations. In particular, we focused on the interplay of two experimentally accessible parameters: nanoparticles' size and the strength of the nanoparticle interaction with the polymer, what we also call affinity (related to the nanoparticle-polymer interface free-energy ${ }^{4 / 28}$ ). In both cases, we find a general, robust behaviour in adhesion strength that does not depend on details of the polymer network, such as its stiffness or its surface tension.

We conclude that nanoparticles should have a size close to the polymer mesh size, and in any case slightly smaller rather than higher. Affinity is also important in that nanoparticles provide a reinforcement only when this is higher than a critical value, but weaken the interface otherwise, a result previously found also for nanoparticle gluing polymer in the collapsed rather than swollen state. ${ }^{4}$ This optimal size comes as the result of two main factors influencing the interfacial reinforcement in an opposite way. On the one side, the smaller are the nanoparticles, the more effective they are at reinforcing the network's interface if confined there, because more polymer-nanoparticle bonds can be established at a fixed surface density. On the other side, however, the smaller the nanoparticles the higher is their ability to diffuse into the bulk, in which case they do not contribute to interface reinforcement. Strictly speaking, our results show the response of the system right after application of the nanoparticles to the interface and a more accurate treatment of aging effects require studying long-term diffusion. The latter will be relevant for all those cases where the particles cannot be kinetically trapped at the interface for times comparable to those required

in applications, which as we show depend on both the nanoparticle size and affinity. Such a study, outside the scope of the present manuscript, will necessarily require some kind of multi-scale modelling as the need to describe the system down to nanoscale details does not allow to access the required time-scales. For example, similarly to what we have done here, 
one could use simulations to estimate the (free-)energy as a function of the distance from the interface as an external field for nanoparticle diffusion (see, e.g., 29130 to study the relevant generalised diffusion equation to generate the correct, time-dependent nanoparticle-density profile before starting the straining procedure.

Finally, we conclude by speculating that our results also suggest that nanoparticles of different shapes might actually provide a better alternative to spherical ones. In particular, two-dimensional nanoparticles might provide more interfacial strengthening because they can be confined to the interface due to their dimensions but still avoiding the depletion of polymer because of their limited volume, thus increasing the number of nanoparticle-polymer contacts necessary for strengthening. Computational studies in these directions are ongoing and will be reported in future publications.

\section{Acknowledgement}

We are grateful to the UK Materials and Molecular Modelling Hub for computational resources, which is partially funded by EPSRC (EP/P020194/1).

\section{Supporting information}

Supporting Information: equilibration protocol, reinforcement model. 


\section{References}

(1) Rose, S.; Prevoteau, A.; Elziere, P.; Hourdet, D.; Marcellan, A.; Leibler, L. Nanoparticle solutions as adhesives for gels and biological tissues. Nature 2014, 505, 382.

(2) Kim, J.-H.; Kim, H.; Choi, Y.; Lee, D. S.; Kim, J.; Yi, G.-R. Colloidal mesoporous silica nanoparticles as strong adhesives for hydrogels and biological tissues. ACS applied materials $\&$ interfaces 2017, 9, 31469-31477.

(3) Kim, S.; Kang, T. H.; Yi, G.-R. The effect of blob size in polymer networks on nanoparticle-mediated adhesion of hydrogels. Soft Matter 2019, 15, 9942-9948.

(4) Molinari, N.; Angioletti-Uberti, S. Nanoparticle Organization Controls Their Potency as Universal Glues for Gels. Nano letters 2018, 18, 3530-3537.

(5) Cao, Z.; Dobrynin, A. V. Nanoparticles as Adhesives for Soft Polymeric Materials. Macromolecules 2016, 49, 3586-3592.

(6) Appel, E. A.; Scherman, O. A. Gluing gels: A nanoparticle solution. Nature materials $\mathbf{2 0 1 4}, 13,231-232$.

(7) Levental, I.; Georges, P. C.; Janmey, P. A. Soft biological materials and their impact on cell function. Soft Matter 2007, 3, 299-306.

(8) Schiller, J. L.; Lai, S. K. Tuning Barrier Properties of Biological Hydrogels. ACS Applied Bio Materials 2020, 3, 2875-2890.

(9) Stevens, M. M.; George, J. H. Exploring and engineering the cell surface interface. Science 2005, 310, 1135-1138.

(10) Molinari, N.; Sutton, A.; Mostofi, A. Mechanisms of reinforcement in polymer nanocomposites. Physical Chemistry Chemical Physics 2018, 
(11) Kim, W. K.; Moncho-Jordá, A.; Roa, R.; Kanduc, M.; Dzubiella, J. Cosolute partitioning in polymer networks: Effects of flexibility and volume transitions. Macromolecules 2017, 50, 6227-6237.

(12) Angioletti-Uberti, S. Theory, simulations and the design of functionalized nanoparticles for biomedical applications: A Soft Matter Perspective. npj Computational Materials $2017,3,48$.

(13) Frenkel, D.; Smit, B. Understanding molecular simulation: from algorithms to applications; Elsevier, 2001; Vol. 1.

(14) Liu, J.; Wu, S.; Zhang, L.; Wang, W.; Cao, D. Molecular dynamics simulation for insight into microscopic mechanism of polymer reinforcement. Physical Chemistry Chemical Physics 2011, 13, 518-529.

(15) Weeks, J. D.; Chandler, D.; Andersen, H. C. Role of repulsive forces in determining the equilibrium structure of simple liquids. The Journal of chemical physics 1971, 54, $5237-5247$.

(16) Arno, M. C.; Inam, M.; Weems, A. C.; Li, Z.; Binch, A. L.; Platt, C. I.; Richardson, S. M.; Hoyland, J. A.; Dove, A. P.; OâĂŹReilly, R. K. Exploiting the role of nanoparticle shape in enhancing hydrogel adhesive and mechanical properties. Nature communications 2020, 11, 1-9.

(17) Plimpton, S. Fast parallel algorithms for short-range molecular dynamics. Journal of computational physics 1995, 117, 1-19.

(18) Hoover, W. G. Canonical Dynamics: Equilibrium Phase-Space Distributions. Phys. Rev. A 1985, 31, 1695.

(19) Hoover, W. G. Constant-Pressure Equations of Motion. Phys. Rev. A 1986, 34, 2499. 
(20) Nosé, S. A Unified Formulation of the Constant Temperature Molecular Dynamics Methods. J. Chem. Phys. 1984, 81, 511-519.

(21) Humphrey, W.; Dalke, A.; Schulten, K. VMD: visual molecular dynamics. Journal of molecular graphics 1996, 14, 33-38.

(22) Yang, L.; Srolovitz, D. J.; Yee, A. F. Extended ensemble molecular dynamics method for constant strain rate uniaxial deformation of polymer systems. The Journal of chemical physics 1997, 107, 4396-4407.

(23) Kim, W. K.; Kanduč, M.; Roa, R.; Dzubiella, J. Tuning the permeability of dense membranes by shaping nanoscale potentials. Physical review letters 2019, 122, 108001.

(24) Allen, M. P.; Tildesley, D. J. Computer simulation of liquids; Oxford university press, 2017.

(25) Hänggi, P.; Talkner, P.; Borkovec, M. Reaction-rate theory: fifty years after Kramers. Reviews of modern physics 1990, 62, 251.

(26) Cukierman, E.; Pankov, R.; Stevens, D. R.; Yamada, K. M. Taking cell-matrix adhesions to the third dimension. Science 2001, 294, 1708-1712.

(27) Lang, N. R.; Münster, S.; Metzner, C.; Krauss, P.; Schürmann, S.; Lange, J.; Aifantis, K. E.; Friedrich, O.; Fabry, B. Estimating the 3D pore size distribution of biopolymer networks from directionally biased data. Biophysical journal 2013, 105, 1967-1975.

(28) Kaelble, D. Peel adhesion: influence of surface energies and adhesive rheology. The Journal of Adhesion 1969, 1, 102-123.

(29) Angioletti-Uberti, S.; Ballauff, M.; Dzubiella, J. Dynamic density functional theory of protein adsorption on polymer-coated nanoparticles. Soft Matter 2014, 10, 7932-7945. 
(30) Angioletti-Uberti, S.; Ballauff, M.; Dzubiella, J. Competitive adsorption of multiple proteins to nanoparticles: the Vroman effect revisited. Molecular Physics 2018, 116, 3154-3163. 